\title{
Políticas de ciencia, tecnología e innovación en México: aproximación a su análisis
}

\author{
Science, Technology and Innovation's Policies in Mexico: approach to analysis \\ Carolina Valero Olmedo ${ }^{a}$, Amelia Molina García ${ }^{b}$ Christian Israel Ponce Crespo ${ }^{c}$
}

\begin{abstract}
:
One of the axes of the international public agenda is the generation of science and technology, as drivers of economic and social development. In Mexico, given the change of the six-year term (2019-2024), the need to deliberately raise a national public agenda on Science, Technology and Innovation of high impact, which allows to favor the economic, social and cultural development conditions of the country. This within the framework of the law and the role of the State for the definition of policies in this area. Derived from the above, this document analyzes the aspects that characterize the definition of public policies, their role in the promotion and development of Science, Technology and Innovation, as well as the possibility of universities in the process of transformation of government action that is more oriented to a governance approach, which includes the active participation of the scientific community and academia.
\end{abstract}

\section{Keywords:}

Science, technology and innovation, public policy

\section{Resumen:}

Uno de los ejes de la agenda pública internacional es la generación de ciencia y tecnología, como impulsores del desarrollo económico y social. En México, ante el cambio de sexenio (2019-2024), se muestra como emergente la necesidad de plantear, de manera deliberativa con otros agentes de la sociedad, una agenda pública nacional en Ciencia, Tecnología e Innovación de alto impacto, que permita favorecer las condiciones de desarrollo económico, social y cultural del país. Esto en el marco del derecho y de la función del Estado para la definición de políticas en este rubro. Derivado de lo anterior, en este documento se analizan los aspectos que caracterizan la definición de las políticas públicas, el papel de estas en la promoción y desarrollo en materia de Ciencia, Tecnología e Innovación, así como la posibilidad de las universidades en el proceso de transformación de la acción gubernamental que se oriente más a un enfoque de gobernanza, donde se incluya la participación activa de la comunidad científica y de la academia.

\section{Palabras Clave:}

Ciencia, tecnología e innovación, políticas públicas

Reporte de investigación recibido: 23 de septiembre de 2019

Dictaminado: 20 de octubre de 2019

Segunda versión: 26 de octubre de 2019

Aceptado: 28 de octubre de 2019

\footnotetext{
a Universidad Autónoma del Estado Hidalgo. Estudiante del Doctorado en Ciencias de la Educación, Email: carovalero28@gmail.com

b Autor de Correspondencia, Universidad Autónoma del Estado de Hidalgo, Área Aacdémica de Ciencias de la Educación, ORCID: https://orcid.org/0000-0002-8268-8421, Email: molinag@uaeh.edu.mx 


\section{INTRODUCCIÓN}

La sociedad actual se enmarca en la globalización, no solo de la economía sino también del conocimiento y la innovación, en el avance desmedido de las tecnologías de la información y comunicación y en la producción científica; la investigación, el desarrollo tecnológico y la innovación adquieren una gran relevancia como impulsores del desarrollo social, lo que agudiza las exigencias de productividad y competitividad de las personas y las organizaciones y arroja resultados dispares entre los países al nutrirse de una dinámica en la que innovación del conocimiento es la última fuente de la generación de valor (Aguilar, 2006).

Las tendencias recientes en las políticas de ciencia y tecnología a nivel mundial, radican en implementar estrategias que orienten hacia la innovación que permitan consolidar el crecimiento económico, la cohesión social y atender retos globales y sociales, como la pobreza, el cambio climático y la salud. Por una parte, la Organización de las Naciones Unidas para la Educación, la Ciencia y la Cultura (UNESCO por sus siglas en inglés), a través de la Agenda 2030, considera fundamental y de forma transversal, la generación de ciencia y tecnología para el logro y alcance de objetivos de desarrollo sostenible, dentro de lo que considera la reducción de la desigualdad, el cuidado y conservación del medio ambiente y estimular el desarrollo económico.

De acuerdo con Loray (2017), las políticas que orientan este campo se ubican básicamente dos enfoques: una perspectiva científico y tecnológica que privilegia el papel de la ciencia y su difusión, como expresión cultural y fuente de conocimientos para el uso productivo y social, las cuales han sido desarrolladas dentro de la UNESCO, la Organización de los Estados Americanos (OEA) y el Banco Interamericano de Desarrollo (BID). La segunda, presenta un enfoque económica-estructuralista, que aduce que el hecho de contar con capacidades propias de investigación científica y desarrollo tecnológico genera las herramientas para apropiarse de los beneficios del aumento de la productividad, la industrialización, el comercio exterior y el crecimiento económico.

En concordancia con esta segunda perspectiva, la Comisión Económica para América Latina y el Caribe (CEPAL, 2008) considera imperante asumir la importancia de la investigación científica para incrementar el patrimonio cultural de la sociedad, formar recursos humanos y generar conocimiento para el desarrollo tecnológico e innovador, que permita avanzar hacia una sociedad basada en el conocimiento, en la que se construyan puentes entre la ciencia y la tecnología y que orienten a mejorar la calidad de vida de la población. Planteamiento que se refuerza con las perspectivas en ciencia, tecnología e inovvación para América Latina de la Organización para la Cooperación y el Desarrollo Económicos (OCDE) (2018), donde refiere al impacto de megatendencias como los cambios sociales, económicos, politicos y tecnológicos a gran escala, como aspectos importantes a tomar en cuenta en la definición de políticas y en la colaboración entre actores como el gobierno, la industria y universidades, así como una mayor inversión económica para la innovación.

Ante tal contexto, México requiere asumir una nueva relación de responsabilidades entre el Estado, los diversos actores de la sociedad civil, el sector privado y la academia, para con ello estar en condiciones de definir nuevas políticas de ciencia, tecnología e innovación, que favorezcan el desarrollo económico, social y cultural del país.

Dicho lo anterior, esta aproximación a la revisión de políticas publicas en materia de ciencia, tecnología e innovación se dirige al análisis documental respecto a los lineamientos sobre las condiciones que posibilitan $u$ obstaculizan la participación incluyente del sector Ciencia, Tecnología e Innovación (CTI) y sus actores en la toma de decisiones para el diseño de la política científica y tecnológica del país. De esta manera, podemos identificar como agentes que representan los diversos niveles de definición de las políticas, a los organismos internacionales en el nivel macro, las instituciones de gobierno federal a nivel meso y, en el plano de lo micro, a las instituciones de educación superior, a las universidades, las empresas, los científicos o académicos dedicados a la ciencia, y a los desarrolladores de tecnología e innovación

Para desarrollar el ejercicio de análisis que se propone, se mantendrá como eje analítico los componentes que caracterizan el enfoque de gobernanza, donde el Estado promueve y permite la participación activa y deliberativa de los diferentes actores del Sistema de Ciencia y Tecnología e Innovación, que darán pauta para trabajos posteriores, en los que se pueda analizar cómo y desde dónde se toman las decisiones en este rubro desde el ámbito gubernamental en México.

El trabajo está integrado por tres partes, en la primera se hace una revisión de lo que son las políticas públicas, para de ahí identificar las que refieren a ciencia, tecnología e innovación; la segunda, refiere a las condiciones en las que se gesta la política de ciencia, tecnología e innovación, y la tercera, que aborda el papel de las universidades como agentes involucrados en la gestión de las políticas de ciencia, tecnología e innovación. Esto para dar paso al desarrollo de conclusiones que se derivan del ejercicio analítico propuesto. 


\section{1.- Ciencia, tecnología e innovación: definiciones conceptuales como políticas públicas}

Las políticas de ciencia, tecnología e innovación, se entienden como "el conjunto de medidas colectivas tomadas por un gobierno con el propósito de impulsar el desarrollo de la investigación científica y tecnológica, así como emplear los resultados de estas investigaciones para alcanzar objetivos políticos" (Salomon, 1997, citado en Loray, 2018: 20). Estas políticas relacionadas directamente con los temas de desarrollo y crecimiento, han sido producto de aspectos históricos y sociales, al definir que el trabajo científico aportaba al logro de los objetivos nacionales, por lo que la calidad de la actividad y sus productos, los medios necesarios para su desarrollo y su orientación, se convirtieron en materia de análisis dentro del conjunto de las políticas públicas y como resultado de acciones del Estado y diversos actores sociales, como las universidades y las empresas bajo el esquema de la Triple Hélice, que pone de manifiesto la necesidad de dicho vínculo como un medio para fomentar las innovaciones y el crecimiento.

De esta forma, el Estado juega un papel importante en la promoción de las políticas de ciencia y tecnología, que radica en la responsabilidad de promover la creación de nuevos conocimientos y la formación de nuevos investigadores que aporten, incluso a la generación de una cultura para la paz. Esto, tomando como referente que es a partir de la culminación de la Segunda Guerra Mundial cuando se comienza a visualizar la relación entre ciencia y gobierno.

Si bien la ciencia y tecnología aparece como un campo específico de las políticas públicas, éstas se dan en función de ciertas demandas del propio Estado, de los intereses académicos y de la ciudadanía preocupada por la capacidad de la insertar a la ciencia en la vida cotidiana. Estos actores, con capacidad de agencia, generan ciertas culturas políticas con diferentes niveles de interacción, dinámicas de participación, e intereses. Elzinga y Jamison (1996) afirman que la ciencia y la tecnología establecen una relación directa con los valores políticos y culturales de los actores, lo que propicia también relaciones de poder que generan conflicto y tensión. De esta forma, los autores proponen cuatro tipos de culturas que influyen en la formulación de políticas de ciencia y tecnología:

a) Burocrática: encarnada en el aparato del Estado, que administra y organiza la ciencia para ponerla a disposición de la política

b) Académica: correspondiente a la comunidad científica, que prioriza y vigila el sentido ético, autónomo y normativo de la ciencia frente a otros intereses. c) Económica: visualizada desde los empresarios y responsables de la política económica pero que se interesan en las aplicaciones tecnológicas e innovadoras de la ciencia.

d) Cívica: encarnada en los movimientos sociales en diferentes temas de la política pública pero que presta atención a las repercusiones de la ciencia en cada uno de ellos.

Dichas culturas se ponen de manifiesto en el diseño e implementación de políticas de ciencia y tecnología, y permea en los diferentes niveles de subjetividad de los agentes, según su nivel de participación en la vida pública pero que repercute en lo personal como estados de consciencia individual y la voluntad de incidencia. Es decir, la motivación, responsabilidad y voluntad de buscar una mejora social.

Para Codner y Del Bello (2011) y Del Bello y Abeledo (2007), citados por Romina Loray (2017:70-71), existen básicamente tres tipos de políticas en relación estricta con los objetivos de las políticas de Ciencia, Tecnología e Innovación: a) horizontales, b) selectivas o sectoriales $y, c)$ de frontera o focalizadas. Las políticas horizontales se fundamentan en fallas del mercado, que constituyen dificultades para la apropiación y asimilación de los conocimientos científicos y tecnológicos generados en innovaciones productivas o sociales. En términos generales, estas políticas incluyen medidas tendientes a apoyar la formación de capital humano, apoyar actividades de producción (certificaciones, control de calidad, entre otras) e incentivos para la infraestructura y el sector empresarial.

Las políticas sectoriales requieren una mayor capacidad institucional, por cuanto involucran sectores o áreas de interés específico, aplicando un amplio conjunto de instrumentos particulares, desde la atracción selectiva de inversión extranjera directa, los incentivos y subsidios para sectores o para la producción específica de actividades y programas de apoyo a la competitividad de las actividades industriales, hasta la producción directa de las empresas estatales y la implementación de contratos públicos (Peres y Primi, 2009, en Lorey, 2017). Finalmente, las políticas de frontera responden a una visión de desarrollo nacional más amplio, y tienen por objeto la creación de capacidades en áreas tecnológicas y científicas estratégicas (Peres y Primi, 2009, en Lorey, 2017).

Este tipo de políticas pueden considerarse a partir de la expresión de estrategias más complejas, que requieren fuertes capacidades de gestión institucional y efectiva coordinación de diferentes grupos de interés.

El origen en la conformación de las políticas públicas en materia de ciencia, tecnologia e innovación inicia cuando concluye la segunda guerra mundial, y a partir de ese momento, como afirma Loray (2017:72), "la ciencia 
quedó vinculada con el poder como nunca lo había estado antes". Además de otros fenómenos, como la creciente importancia que fue adquiriendo el conocimiento científico y tecnológico, la emergencia de la Big Science y el papel que fue desempeñando el Estado en la gestión de las actividades de investigación en los países industrializados. Todos ellos, fenomenos que afianzaron el el rol del Estado en la definición de las políticas públicas en materia de Ciencia Tecnología e Innovación.

Mientras en los paises industrializados se vivía, durante la década de los setenta del siglo pasado, un importante desarrollo en materia de ciencia y tecnología, en los países latinoamericanos en cambio, se producía un profundo deterioro de la estructura productiva y del sistema cientifico y tecnológico. Situación que obligó a la clase política latinoamericana a implementar medidas profundas para restructurar los Sistemas Nacionales de Ciencia, Tecnología e Innovación y fue hasta finales de los ochenta que se incorporó el concepto de innovación en las estructuras y el diseño de políticas públicas (Albornoz, 2007 en Loray, 2017: 72). De esta manera, la planificación de Ciencia Tecnología e Innovación fue incorporando la variable "innovación" y con ello también, los marcos teóricos para enfocar el proceso de cambio desde una perspectiva de sistemas de innovación.

Es así, que las políticas públicas se entienden como "el conjunto de acciones resultantes de un entramado institucional y de actores, tanto públicos como privados, que participan en la atención de un problema público" (Treviño, 2013: 152). En términos de Ozlak y O’Donnell (1995), las políticas públicas constituyen un conjunto de iniciativas y respuestas, manifiestas o explícitas que permiten inferir la posición predominante del Estado frente a unas cuetiones que ateñen a sectores significativos de la sociedad. Como campo de estudio, en las últimas décadas, han adquirido una gran importancia en el área de las ciencias sociales contemporáneas, en el sentido de alcanzar un marco conceptual común y entender su surgimiento y evaluación de forma tal que permitan promover una mayor eficacia y democracia en la toma de decisiones que afectan de manera directa a diversos sectores de la sociedad (Méndez, 2000).

Su diseño, implementación y evaluación ha transitado históricamente desde dos enfoques: el administrativo y el gerencial. En el administrativo, se entendía a las políticas públicas como racionalización basada en procesos burocráticos, donde Laswell (Aguilar, 2000), en la década de los cincuenta, propuso la necesidad de mayor conocimiento por parte del gobierno en sus decisiones públicas para aumentar la racionalidad de las políticas. El enfoque gerencial, en cambio, entendía a las políticas públicas basadas en la gestión y la toma de decisiones, en la que se buscaba la satisfacción de los clientes (ciudadanos), desde una perspectiva más empresarial que promueve la flexibilización de los procesos administrativos, modificando así los sistemas burocráticos del Estado tradicional Weberiano.

Dentro de este nuevo enfoque de gestión, los clientesactores son considerados como agentes activos en la toma de decisiones para la política pública; se introduce el término de gobernanza, entendida no sólo como una forma de gobernabilidad sino también como participación conjunta entre los ciudadanos y los gobiernos, en donde los diversos actores hacen valer su derecho de participación social y limita las funciones del Estado a la ejecución de actividades técnicas relacionadas con el mantenimiento del orden social (Gómez, 2012).

Es decir, la gobernanza constituye un mecanismo de colaboración en la política pública, que surge al tiempo que lo hace un ciudadano que exige y hace valer sus derechos y ejerce la soberanía de la política por sobre la técnica de las políticas (Prats, 2005). Es por ello, que la propuesta de la gobernanza no sólo es una reforma administrativa del Estado, sino que supone, también, cambios políticos profundos en la forma de entender la relación entre el Estado y las sociedades (Aguilar, 2010). Dicho enfoque considera, de manera implícita, el conocimiento de las relaciones de poder que enmarcan al Estado, pero también el interés de la sociedad por ser partícipe en la creación e implementación de políticas públicas. La participación de la ciudadanía en las decisiones políticas se visibiliza como una forma de intervención de los individuos en actividades públicas, en las que estos poseen y representan los intereses de una determinada sociedad, que les lleva a generar, desde la esfera pública, una relación con el Estado.

Lo anterior permite a la vez, la definición del enfoque de redes, en el que se consideran diversas estrategias de intervención gubernamental, sustentadas en las relaciones y vínculos con otros múltiples actores de la esfera pública. Esta relación puede darse en diversas dimensiones jerárquicas: de forma horizontal, en las que se pone énfasis en los niveles y estructuras de relación entre los actores que componen la red; de forma vertical, que está dada por el proceso de diferenciación entre los actores según su nivel de jerarquía, permitiendo con ello los procesos de distribución de poder estructural y por ende, de ordenación jerárquica respecto a la intervención gubernamental, esta dimensión, tambien permite relaciones interinstitucionales entre diferentes redes de política pública o sectores y ámbitos de gobierno (Vázquez 2014: 153).

Este nuevo enfoque de redes, se complementa con un sistema deliberativo, que se centra en las interacciones de los actores en los procesos de estructuración y decisión de políticas públicas, supone que las partes involucradas alcancen consensos sobre los temas 
de interés público, bajo la lógica de acuerdos racionalmente motivados (Habermas, 1998, en Garrido et al., 2016), en donde, de acuerdo con la teoría habermasiana, la interacción es la base para un verdadero cambio social en las sociedades modernas, lo que conlleva a la implementación de mecanismos que aseguren formas de participación ciudadana, posibiliten la integración sociopolítica de la ciudadanía y otorguen legitimidad a las decisiones políticas.

\section{2.- Condiciones de la política de ciencia, tecnología e innovación}

A partir de los referentes mencionados, en cuanto a que el desarrollo económico, social y cultural de los países y de las personas depende, en gran medida, del desarrollo de la ciencia, la tecnología y la innovación, cobra especial atención el concepto de globalización, el cual refiere al fenómeno que permea la relación estrecha que existe en las interacciones en el mundo contemporáneo, caracterizado por el acelerado desarrollo tecnológico, y por las nuevas formas de intervención de la opinión pública, que influyen en la forma en que el Estado toma decisiones en los temas de economía, cultura, políticas y aspectos sociales, los cuales anteriormente sólo eran de competencia exclusiva del propio Estado.

En este sentido, los organismos internacionales y los grupos financieros como la Organización de las Naciones Unidas para la Educación, la Ciencia y la Cultura (UNESCO), la Organización para la Cooperación y el Desarrollo Económico (OCDE), el Banco Interamericano de Desarrollo (BID), la Organización de Estados Americanos (OEA), entre otros, influyen en la toma de decisiones en materia de política pública, posicionando al Estado como un mediador para su gestión en la sociedad.

Dicho fenómeno de globalización, no sólo ha promovido el avance y desarrollo tecnológico, sino que también ha sido causal de la generación de un estado de competencia y de grandes brechas de desigualdad, lo que representa por ende, un menor nivel de desarrollo económico, social y cultural de los países en desventaja, y con relación al desarrollo de ciencia y tecnología.

Dado lo anterior, el reto del Estado es asegurar el diseño e implantación de políticas que consideren una gestión inversa a la que se desarrolla en la actualidad, es decir, una gestión que parta del contexto micro, con sus demandas y necesidades específicas, direccionadas hacia un contexto macro.

En el caso de México, los primeros 70 años del siglo XX, representaron el inicio de la toma de decisiones en materia de política pública en ciencia y tecnología, aunque estas representaban fines aplicativos y de corto alcance, principalmente en temas de agricultura e industria. Sin embrago y de acuerdo con Pérez-Tamayo
(2010), estas políticas dieron pocos frutos principalmente porque no se trataron de decisiones con alta prioridad y visión a largo plazo, sino más bien como medidas de carácter simbólico y que se tomaron al margen de la comunidad científica, aunado a que el Estado no consideraba que la ciencia contribuyera a sus dos intereses primordiales: el control político y el desarrollo económico.

A finales de 1970 se crea el Consejo Nacional de Ciencia y Tecnología, como órgano central de la política científica, y es el medio a través del cual el gobierno asigna recursos a la ciencia, además de que representa a la comunidad científica del país. Sin embargo, es a partir de la década de los noventa, que comienzan a promoverse importantes cambios políticos en materia de investigación y desarrollo; se establece un incremento en el gasto del Producto Interno Bruto (PIB) destinado a la investigación, pasando del $0.28 \%$ en 1990 al 0.35 en 1995. Lo que trajo como resultado una mejora importante en los indicadores de ciencia y tecnología, comenzando por incrementar el número de científicos y publicaciones en el Science Citation Index (Ibarra, 2010). En este sentido, México ha incrementado, en las últimas dos décadas, la inversión (alcanzando el $0.5 \%$ del PIB). No obstante, esta cifra aún está por debajo de las que presentan países desarrollados (Banco Mundial, 2019), lo que detona que la contribución del país, a la producción de conocimiento científico y tecnológico, aún no alcance el uno por ciento y que su impacto en este campo sea deficiente, pues de acuerdo con el CONACyT (2016), es atribuible a los siguientes aspectos:

a) La población económicamente activa que se dedica a la investigación y formación de recursos humanos, representa solo la décima parte de cada mil en comparación con paises más avanzados.

b) El factor de impacto a nivel mundial de las publicaciones en México, en el periodo 20122016, en comparación con países miembros de la OCDE fue de 0.98 , ocupando el lugar 38.

c) El número de patentes, para el mismo periodo, decreció en 7.29 por ciento (de 9,338 a 8657), lo que corresponde al 4.92 por ciento en relación con países como Alemania o Japón que tienen 7.54 y 6.54 por ciento respectivamente

El desarrollo de la ciencia en el país ha sido aleatorio por múltiples factores, como la falta de asignación de recursos, lo que da cuenta, en palabras de PérezTamayo (2010), de la indiferencia del Estado por la potencialización del desarrollo científico y tecnológico, como contraparte del basto interés de la comunidad científica en tal desarrollo.

De igual forma, existen condiciones estructurales en México que imposibilitan el desarrollo de la ciencia y la 
tecnología, comenzando porque los grandes retos que permean la agenda nacional en materia de política pública en este ámbito recaen, a juicio de los propios científicos, en el financiamiento, el aseguramiento de la calidad, la comercialización, protección y utilización de los conocimientos científicos y tecnológicos, la comunicación científica, la formación de los recursos humanos y la internacionalización del conocimiento científico (Martínez-Domingo, 2011; Didou, Góngora, Durand, Pineda y Badillo, 2013).

Actualmente, el Plan Nacional de Desarrollo 2018-2024, hace referencia a que "el gobierno federal promoverá la investigación científica y tecnológica; apoyará a estudiantes y académicos con becas y otros estímulos en bien del conocimiento. El CONACYT coordinará el Plan Nacional para la Innovación en beneficio de la sociedad y del desarrollo nacional con la participación de universidades, pueblos, científicos y empresas" (PND, 2019: 58).

Bajo un enfoque de gobernanza, que hace alusión a la participación de la ciudadanía, que incluye cualquier forma de acción colectiva, de reivindicación 0 de respuesta a las convocatorias formuladas desde el gobierno para incidir en las decisiones de política pública (Díaz, 2017), el gobierno federal marca la pauta para la organización en conjunto con otros agentes sociales. Sin embargo y desde la percepción de la comunidad científica (FCCyT, 2019), además de las modificaciones a las leyes y políticas en este campo, la toma de decisiones se manifiesta de manera unilateral y es caracterizada como estadocentrismo.

Es decir, que ese sentido estadocentrista, como un agente que ejerce control autoritario de aparatos burocráticos y una movilización de reglamentaciones que establecen un marco y limitaciones a los actores, hace visualizar al Estado (que se considera) como el único en instalarse en el centro de las decisiones públicas, definiendo políticas autoritarias acciones ejecutivas que influyen o determinan la conducta al interior del Sistema (Sáenz y Molina, 2017), lo que a la vista de la comunidad científica y las organizaciones nacionales inmersas en el desarrollo de ciencia y tecnología, representa que el Estado delegue, en el Consejo Nacional de Ciencia y Tecnología, la responsabilidad del diseño, implementación y evaluación de políticas destinadas a este rubro, sin considerar a otros agentes de la sociedad que requieren y buscan estar involucrados en la toma de decisiones.

\section{3.- Las universidades como agentes involucrados en la gestión de las políticas de ciencia y tecnología}

De acuerdo con Armando Alcántara (2000), las universidades son instituciones centrales en las sociedades modernas, ya que proporcionan la educación que requieren las economías basadas en el desarrollo tecnológico, se constituyen como los centros más importantes para la investigación y la innovación en diferentes áreas, y son participantes activos del sistema internacional de conocimientos.

De esta manera, las universidades aseguran que la sociedad tenga consciencia de lo que sucede en el mundo cada vez más globalizado de la ciencia, el trabajo académico y la investigación. En este mismo sentido, la universidad, como institución productora de conocimientos y formadora de opinión y tendencias tiene una responsabilidad social incuestionable (Carrizo, 2004); es el espacio donde se fomenta la producción de conocimiento científico pertinente y útil a las exigencias de nuestro tiempo, ya que de acuerdo con Acosta (2013), la emergencia y consolidación de la sociedad del conocimiento, han generado que esta última cobre un alto valor en los ámbitos de la vida social y que se constituya como un desafío a superar en las IES, principalmente en las universidades.

En ese sentido y de acuerdo con Gibbons (1998), las Instituciones de Educación Superior, entre ellas las universidades, tienen grandes retos ante los cambios actuales en la producción del conocimiento, empezando por la necesidad de una profunda transformación epistemológica y una renovadora corriente ética y democrática del conocimiento (Carrizo, 2004), aunado a que sus formas de organización fungen de acuerdo con las estructuras disciplinares, las cuales también están sufriendo cambios, principalmente orientados hacia la interdisciplinaridad y el impacto social de la generación científica.

Dichos cambios, requieren a su vez, del diálogo entre diversos saberes en el campo de las ciencias, entre diversos implicados en la cadena de producción científica, como el actor político y la interacción con otros productores de conocimiento, como el sector industrial en un contexto de aplicación y configurado por un conjunto diverso de demandas intelectuales y sociales (Sánchez, et al. 2013), con la finalidad de que dicha producción pueda usarse con eficacia en el contexto que requiere la solución de problemas específicos; y por otro lado, su papel fundamental en la formación de recursos humanos calificados, así como en la generación de ciencia y tecnología como parte del aporte al desarrollo científico del país. 
Desde los planteamiento que anteceden, diversos autores (Gibbons, 1998; Santos, 2005; Sánchez, et al. 2013) refieren que la universidad debe reconfigurar el sentido de sus funciones sustantivas: docencia, investigación y extensión, y que la generación de conocimiento, signado por la coyuntura de las demandas del capitalismo globalizado, generan tensiones respecto a su orientación y su ritmo de producción, incluso su aplicabilidad o descontextualización; por ende, genera también tensiones en aspectos burocráticos que tienen repercusiones en el desempeño de la labor investigativa de los profesores, y que de acuerdo con Martínez (2011), empiezan a ser un síntoma característico de las Instituciones de Educación Superior.

Esto último, es analizado por Christian Ponce-Crespo (2017), desde el concepto de condiciones de producción intelectual, donde lo institucional genera dos tipos de disposiciones: las estructuradas, que refieren a los mecanismos de vigilancia y control, de normatividad, formas de organización académica y procesos de gestión, que son asignados a los académicos, lo que a nivel institucional se refleja en un aumento y diversificación en los roles, actividades y responsabilidades.

Por otro lado, las disposiciones estructurantes, que en términos de Ponce (2017), son principios generadores de prácticas y representaciones, son duraderas y transferibles, y se reflejan en el actuar cotidiano de los académicos. Es decir, de la manera en cómo se enfrentan a las condicionantes de la estructura institucional, construyen significados y se externalizan a partir de las propias prácticas, formas de respuesta, asimilación, adaptación y o resistencia (Ponce, 2017).

\section{Conclusiones}

Ante los nuevos retos de la sociedad globalizada y en el marco del desarrollo científico y tecnológico, la definición de las políticas públicas en este rubro, requiere un nuevo enfoque de participación de los diversos agentes, cuyos intereses y motivaciones permean la definición de la agenda nacional.

En esta participación, será de gran importancia la academia, representada por las universidades, cuyo compromiso social promueve la formación de nuevos investigadores y tecnólogos con la más alta calidad académica. Otro agente de participación lo constituye el Estado mexicano, como el encargado de generar los vínculos y favorecer las condiciones de estructura que promuevan el desarrollo científico y tecnológico del país, en el entendido del esquema de competencia global, en el que México ya se encuentra inmerso y comprometido con el desarrollo sostenible que plantean las agendas públicas internacionales.
Destaca, para su implementación, el enfoque basado en la gobernanza y la toma de decisiones con y para la ciudadanía, donde como nuevo esquema, se promueve la participación de los diversos actores involucrados en el diseño, implementación y evaluación de las políticas públicas, tomando en cuenta principalmente, las necesidades de la sociedad. Lo anterior, con una visión deliberativa de la participación de otros agentes, lo que implicaría trocar la toma de decisiones, de una visión burocrática a una visión más democrática y participativa. No obstante lo anterior, es importante reconocer que en México interactúan los dos enfoques: el burocrático y el centrado en la administración, donde prevalece éste último, y los procesos burocráticos siguen estando presentes en la gestión pública. Ante esto, las políticas de ciencia y tecnología se deciden de forma unilateral, sin tomar en cuenta a la academia y lo que dictan los organismos supranacionales, lo que se contrapone con "tipo ideal" (en el sentido weberiano), donde otros agentes, aparte del Estado, legitiman la construcción y funcionamiento de dicho sector, desde un marco normativo de derecho a la participación, el reconocimiento y el desarrollo individual y social.

\section{REFERENCIAS BIBLIOGRÁFICAS}

Acosta, Wilson. (2013) "Modo 3 de producción del conocimiento: implicaciones para la universidad de hoy". Revista Universidad de la Salle (En línea) No. 6 Disponible en: https://revistas.lasalle.edu.co/index.php/ls/article/view/2439/ $\underline{2156}$

Aguilar, Luis. (2000). Problemas públicos y agenda de gobierno. México: Miguel Ángel Porrúa

Aguilar, Luis. (2006). Gobernanza y gestión pública. México: Fondo de Cultura Económica: México

Alcántara, Armando. (2000). "Ciencia conocimiento y sociedad en la investigación científica universitaria". Perfiles Educativos (En línea) No. 87. Instituto de Investigaciones sobre la Universidad y la Educación. México: UNAM. Disponible en http://www.redalyc.org/pdf/132/13208703.pdf

Banco Mundial, (2019). Gasto en Investigación y Desarrollo (\% del PIB). Banco Mundial. Disponible en: http://www.5.bancomundial.org/investigacion/

Carrizo, Luis. (2004). "Producción de conocimiento y políticas públicas". Desafío de la universidad para la gobernanza democrática. Reencuentro (En línea) No. 40. México: UAMXochimilico. Disponible en: http://www.redalyc.org/articulo.oa? $\mathrm{id}=34004010$

CEPAL, (2008). Generación y protección del conocimiento: propiedad intelectual, innovación y desarrollo económico. Canadian International Development Agency. (En línea). Disponible en: https://www.cepal.org/es/publicaciones/2873-generacionproteccion-conocimiento-propiedad-intelectual-innovaciondesarrollo

CONACyT (2014-2018). Programa Especial de Ciencia y Tecnología. Consejo Nacional de Ciencia y Tecnología. (En línea) México: CONACyT. Disponible en: http://www.siicyt.gob.mx/index.php/normatividad/nacional/p rograma-especial-de-ciencia-tecnologia-e-innovacion- 
peciti/2014-programa-especial-de-ciencia-tecnologia-einnovacion/623-peciti-2014-2018/file

CONACyT (2016). Informe General del Estado de la Ciencia, la Tecnología y la Innovación. México 2016. (En línea) México: Consejo Nacional de Ciencia y Tecnología. Disponible http://www.siicyt.gob.mx/index.php/transparencia/informesconacyt/informe-general-del-estado-de-la-ciencia-tecnologiae-innovacion/informe-general-2016/3835-informe-general2016/file

Didou, Sylvie, Góngora, Edgar, Durand, Juan, Pineda, Yvonne y Badillo, Jessica (2013). "La investigación sobre las políticas de ciencia y tecnología. ¿Un tema emergente para la investigación educativa en México?”. En Maldonado, Alma. Educación y ciencia: políticas y producción de conocimiento 2002-2011. México: COMIE-ANUIES.

Elzinga, Aant y Jamison, Andrew. (1996). "El cambio de las agendas políticas en ciencia y tecnología". Revista Zona Abierta (75/76). (En línea) España. Disponible en: http://docs.politicascti.net/documents/Teoricos/ELZINGA_J AMISON.pdf

FCCyT (2019). "Pronunciamiento sobre la iniciativa de ley de humanidades, ciencias y tecnologías". Foro Consultivo de Ciencia y Tecnología. Disponible en: https://www.foroconsultivo.org.mx/FCCyT/boletines-deprensa/pronunciamiento-sobre-la-iniciativa-de-ley-dehumanidades-ciencias-y-tecnolog\%C3\%ADa

Garrido-Vergara, Luis, Valderrama, Luz y Ríos, Javier. (2016). "Democracia deliberativa, instituciones y participación ciudadana en América Latina". Política, (En línea) No.54 Vol. 2), Disponible http://www.redalyc.org/comocitar.oa?id=64551061010

Gibbons, M. (1998). Pertinencia de la Educación Superior en el Siglo XXI. Conferencia Mundial sobre la Educación Superior. París: UNESCO y Banco Mundial.

Ibarra, José. (2010). "La ciencia mexicana ante los desafíos de la globalización: innovación y competitividad para trascender". Revista Ciencia. (En línea) Disponible en: http://www.revistaciencia.amc.edu.mx/online/6851.pdf

Loray, Paola. (2018). Organismos internacionales y políticas de ciencia, tecnología e innovación. El rol del Banco Interamericano de Desarrollo en el diseño e implementación de los Fondos de Innovación Tecnológica Sectorial de Argentina (2009-2015). (Tesis doctoral). Universidad Nacional de Quilmes, Bernal, Argentina. Disponible en: http://ridaa.unq.edu.ar/handle/20.500.11807/819

Loray, Romina. 2017. "Políticas públicas en ciencia, tecnología e innovación: tendencias regionales y espacios de convergencia”. Revista de Estudios Sociales 62: 68-80. Disponible en https://revistas.uniandes.edu.co/doi/full/10.7440/res62.2017. $\underline{07}$

Martínez, Domingo. (2011). Reseña de libro: "Sylvie Didou y Eduardo Remedi. De la pasión a la profesión: investigación científica y desarrollo en México (2008)". México: Casa Juan Pablos. Revista Estudios Sociológicos de El Colegio de México .Vol. XXIX No. $87 . \quad$ Disponible en: http://estudiossociologicos.colmex.mx/index.php/es/article/vi ew/179/179

Méndez, José. (2000). Lecturas básicas de administración y políticas públicas. México: Colegio de México A.C.

OCDE (2018). Perspectivas de la OCDE en Ciencia, Tecnología e Innovación en América Latina 2016. (En Línea). Microsoft Latin América. Disponible en: https://www.oecdilibrary.org/docserver/9789264303546- es.pdf?expires $=1568662586 \&$ id $=$ id\&accname $=$ guest \&checks um=05F5679D685F63F2097B91E9F84A9005

OCDE. (2014). Perspectivas de la OCDE sobre ciencia, tecnología e industria. Informe Iberoamericano. OECD. Disponible en: http://www.oecd.org/publications/perspectivas-de-la-ocdesobre-ciencia-tecnologia-e-industria-2014-version-abreviada9789264226487-es.htm

Oszlak, Oscar y O`Donnell, Guillermo. (1995). Estado y políticas estatales en América Latina: hacia una estrategia de investigación. Revista Redes (En línea) No 4. Argentina: Editorial de la UNQ. Disponible en http://e-tcs.org/wpcontent/uploads/2017/03/Oszlak-ODonnell-1984-Estado-ypol\%C3\%ADticas-estatales-en-América-Latina-hacia-unaestrategia-de-investigación.pdf

Pérez-Tamayo, Ruy. (2010). El Estado y la ciencia en México: Pasado, presente y futuro, pp. 319-350, en: Fix-Zamudio, Héctor y Valadés, Diego. Formación y perspectivas del Estado en Méxcio. Serie Estudios Jurídicos Núm.164. Instituto de Investigaciones Jurídicas. México: UNAM. Disponible en https://archivos.juridicas.unam.mx/www/bjv/libros/6/2873/1 7.pdf

PND. (2019) Plan Nacional de Desarrollo 2019-2024. Disponible en: https://www.dof.gob.mx/nota detalle.php?codigo $=5565599$ $\&$ fecha $=12 / 07 / 2019$

Ponce, Christian Israel. (2017). Las identidades Científicas en la Universidad Autónoma del Estado de Hidalgo. Un análisis comparativo entre dos institutos. Tesis de Doctorado en Pedagogía. México: Universidad Nacional Autónoma de México.

Prats, Joan. (2005) De la burocracia al management, del management a la gobernanza. Madrid, España: Instituto Nacional de Administración Pública.

Sáenz, José A. y Molina, Amelia. (2017). Jornada Escolar Extendida, dos experiencias de gestión escolar en América Latina: Chile y México. Revista Latinoamericana de Políticas y Administración de la Educación (7). Disponible en: http://relapae.com.ar/wpcontent/uploads/relapae 4 4 saenz molina_jornada extendi da.pdf

Sánchez, Luis, Lladó, Dora, Guzmán, Teresa, Pérez, Ricardo, Guzmán, Josefina y Gómez, Margarita. (2013) "Modos colectivos de producción del conocimiento de los académicos de las universidades públicas mexicanas". XIII Coloquio Internacional sobre Gestión Universitaria en las Américas. Disponible https://repositorio.ufsc.br/handle/123456789/114936?show=f $\underline{\text { ull }}$

Treviño, Ernesto, Olivier, María, y Alcántara, Armando. (2013). "La investigación sobre las políticas de la Educación Superior. Un balance de la producción académica generada en México entre los años 2002 y 2012”. En Maldonado, Alma (Coord.), Educación y Ciencia: políticas y producción del conocimiento 2002-2011. México: ANUIES.

Vázquez, Cristo (2014). "Gobernanza y redes de política pública. Un estudio de la vinculación entre gobierno, actores públicosociales y privadas en un área local turística", Revista Mexicana de Análisis Político y Administración Público, (En línea) Vol. 3, núm.1. Disponible en: http://www.remap.ugto.mx/index.php/remap/article/downloa $\underline{\mathrm{d} / 73 / 67}$

\section{NOTAS}

[1] Este reporte parcial de investigación, corresponde a una reelaboración de contenidos que forman parte de una Tesis del programa de Doctorado en Ciencias de la Educación (en proceso) de la Universidad Autónoma del Estado de Hidalgo. 